\title{
The Nuclear Region of a Megamaser in a Southern AGN, NGC 5793: Parsec-Scale Structure and OH Absorption
}

\author{
Y. Hagiwara \\ The Graduate University for Advanced Studies, NRO, Minamimaki-mura, \\ Minamisaku-gun, Nagano 384-13
}

R. Kawabe \& N. Nakai

Nobeyama Radio Observatory, Minamimaki-mura, Minamisaku-gun, Nagano $384-13$

P.J. Diamond

National Radio Astronomy Observatory, Socorro, NM, U.S.A.

\begin{abstract}
We have carried out VLBA observations of the $\mathrm{OH}$ absorption lines toward the nucleus of the active galaxy, NGC 5793 , which is known to have a megamaser in its center. We have also successfully made its continuum image at the $L$ band. It reveals the typical "core-jet" structure on parsec scales. Uniform values of optical depth at each point against the background radio continuum source shows that the column densities of the $\mathrm{OH}$ absorbing cloud uniformly distributes around the radio compact nucleus.
\end{abstract}

\section{Introduction}

NGC 5793 is an edge-on spiral with dimensions of $3 \times 1 \operatorname{arcmin}^{2}$. H I and $\mathrm{OH}$ absorptions against a background radio nucleus were found (Jenkins 1983; Whiteoak \& Gardner 1987). Both absorption lines obtained with VLA revealed plentiful absorbing material in front of the active nucleus. The $21 \mathrm{~cm}$ continuum emission observed with European VLBI network showed that the radio nucleus consists of a bright continuum source whose diameter is about 0.016 arcsec (Gardner et al. 1992), corresponding to $5 \mathrm{pc}$ at the distance of D $=50 \mathrm{Mpc}$ (assuming $\mathrm{H}_{0}=75 \mathrm{~km} \mathrm{~s}^{-1} \mathrm{Mpc}^{-1}$ ). The luminous and compact nucleus has peak brightness temperature of $10^{9} \mathrm{~K}$, giving evidence for the existence of an AGN at the center. In addition to it, we have newly discovered the water maser emisson from the nucleus of the galaxy, which consists of two strong high-velocity features, that are almost symmetrically offset by about $240-260 \mathrm{~km} \mathrm{sec}^{-1}$ from the systemic velocity of the galaxy (Hagiwara et al. 1997). From these results, we suspect that masers originate from the rotating disk within several to $10 \mathrm{pc}$ from the center by analogy with the masing disk of NGC 4258 (Miyoshi et al. 1995).

\section{Results \& Summary}

We observed $\mathrm{OH}$ absorptions in NGC 5793 with the VLBA plus the phased VLA on 1996 November 30 for a duration of 8 hrs. Because of the weak peak flux density of the $\mathrm{OH}$ absorption, the phased VLA was critical to the observations. The $\mathrm{OH} 1665 / 1667 \mathrm{MHz}$ main-line transitions were detected but not resolved. Figure 1 shows the continuum image and Table 1 gives the parameters of the $\mathrm{OH}$ absorption $(1667 \mathrm{MHz})$ seen by the synthesized interferometer beam. 


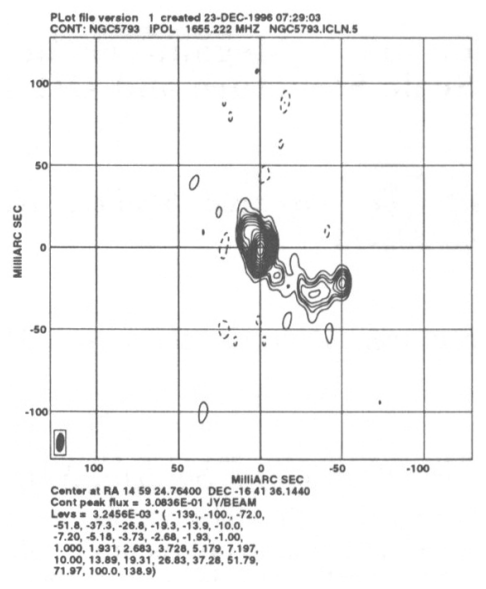

Figure 1. The VLBA continuum map of $1.6 \mathrm{GHz}$.

We successfully obtained the resolved continuum image of $1.6 \mathrm{GHz}$ for the first time, showing the complex milliarcsecond structure with jets. The optical depths of foreground absorptions are uniform at each place against the background continuum structure, resulting in the fact that the cloud causing absorption uniformly covers the background compact continuum nucleus. The presence of the $\mathrm{OH}$ molecule absorption is consistent with the existence of water maser sources in the nuclear region.

Table 1. Parameters of NGC 5793

\begin{tabular}{|c|c|c|}
\hline Parameters $(1667 \mathrm{MHz})$ & VLBA & $V_{L A}{ }^{1}$ \\
\hline Peak optical depth & $0.08 \pm 0.01$ & 0.06 \\
\hline Velocity width ${ }^{2}\left(\mathrm{~km} \mathrm{~s}^{-1}\right)$ & $28.4 \pm 0.5$ & 39.3 \\
\hline
\end{tabular}

Notes: 1: Gardner et al. 1986; 2: FWHM

Acknowledgments. The author thanks the IAU organizing committee for travel support. The National Radio Astronomy Observatory is a facility of the National Science Foundation, operated under a cooperative agreement by Associated Universities, Inc.

\section{References}

Gardner, F. F., \& Whiteoak, J. B. 1986. MNRAS, 221, 537-542.

Gardner, F. F., et al. 1992. MNRAS, 258, 296-300.

Hagiwara, Y., et al. 1997. PASJ, 49, 171-177.

Jenkins, C. R. 1983. MNRAS, 205, 1321-1327.

Miyoshi, M., et al. 1995. Nature, 373, 127-129.

Whiteoak J. B., \& Gardner, F. F. 1987. Proc. Astro. Soc. Aust., 7, 88-91. 